\title{
Preoperative planning and surgical technique for optimizing internal fixation of posterior malleolar fractures: $\mathrm{CT}$ versus standard radiographs
}

Ezequiel Palmanovich ${ }^{1 *}$, Nissim Ohana ${ }^{1}$, Eyal Yaacobi ${ }^{1}$, David Segal ${ }^{1}$, Hetsroni Iftach ${ }^{1}$, Zachary T. Sharfman ${ }^{2}$, Matias Vidra ${ }^{3}$ and Ran Atzmon ${ }^{4}$

\begin{abstract}
Background: A proper reduction and internal fixation of posterior malleolar fractures can be challenging, as intraoperative fluoroscopy often underestimates the extent of the fracture. Our aim was to assess the value of a modified classification system for posterior malleolar fractures, which is based on computed tomography (CT) images, optimizing screw trajectory during fluoroscopic-guided surgery, and to compare it to the Lauge-Hansen classification system to the CT-based classification.
\end{abstract}

Methods: A retrospective review of all ankle fracture operations from January 2014 to December 2016 was performed. Fractures were included if a CT scan was performed within 1 week of the surgery, and the posterior malleolar fragment occupied one third or more of the antero-posterior talar surface or jeopardize the ankle stability. Eighty-five adult ankle fractures with posterior malleolar fragments were included in this study. Fractures were categorized into one of three types, namely "postero-lateral," "postero-medial," or "postero-central," according to the location of the fracture fragment on axial CT image. An optimal trajectory angle for a single-lag screw fixation was measured on the CT cut between a central antero-posterior line and the line intersecting the posterior fragment perpendicular to the major fracture line. Mean trajectory angles were calculated for each fracture type. Fractures were also categorized according to the Lauge-Hansen system.

Results: The mean trajectory angle was $21^{\circ}$ lateral for "postero-lateral" fragments, $7^{\circ}$ lateral for "postero-central" fragments, and $28^{\circ}$ medial for "postero-medial" fragments ( $p<0.01$ for comparisons among the groups). The range of trajectory angles within each group was about $10^{\circ}$, as compared to about $20^{\circ}$ within each Lauge-Hansen type. There were no differences in trajectory angle among the Lauge-Hansen groups ( $p>0.05$ for all comparisons).

Conclusions: There are 3 distinct anatomic subgroups of posterior malleolar fragments, each with an ideal screw trajectory that needs to be used in order to achieve an optimal reduction and fixation.

Keywords: Preoperative planning, Surgical technique, Posterior malleolar fracture, Computed tomography, X-ray

\footnotetext{
* Correspondence: ezepalm@gmail.com

'Orthopedic Department, Meir Hospital, Sapir Medical Center affiliated with the Sackler Faculty of Medicine, Tel Aviv University, 56 Tchernichovsky St, Kfar Saba, Israel

Full list of author information is available at the end of the article
}

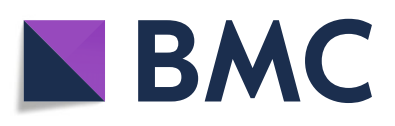

( ) The Author(s). 2020 Open Access This article is licensed under a Creative Commons Attribution 4.0 International License, which permits use, sharing, adaptation, distribution and reproduction in any medium or format, as long as you give appropriate credit to the original author(s) and the source, provide a link to the Creative Commons licence, and indicate if changes were made. The images or other third party material in this article are included in the article's Creative Commons licence, unless indicated otherwise in a credit line to the material. If material is not included in the article's Creative Commons licence and your intended use is not permitted by statutory regulation or exceeds the permitted use, you will need to obtain permission directly from the copyright holder. To view a copy of this licence, visit http://creativecommons.org/licenses/by/4.0/. The Creative Commons Public Domain Dedication waiver (http://creativecommons.org/publicdomain/zero/1.0/) applies to the data made available in this article, unless otherwise stated in a credit line to the data. 


\section{Background}

Ankle fractures are among the most common types of fractures treated by orthopedic surgeons [1]. The incidence of ankle fractures is reported to be 187 fractures per 100,000 people each year [2]. Sixty to $70 \%$ of these injuries are unimalleolar fractures, and 15 to $20 \%$ are bimalleolar fractures involving both the medial and lateral malleoli. Seven to $12 \%$ of all ankle fractures can be classified as trimalleolar fractures, which involve the posterior malleolus in addition to the medial and lateral malleoli $[3,4]$. A posterior malleolar fracture constitutes 7 to $44 \%$ of all ankle fractures [3,5-7]. While less than $1 \%$ of ankle fractures are isolated posterior malleolar fractures, the majority of this fracture commonly occurs in relation to lateral or medial malleolar fractures $[1,8]$. Indications for surgery in cases of posterior malleolar fractures are subject to ongoing debate $[9,10]$. Typically, the size of the posterior malleolar fragment is taken into account when considering operative fixation. Some investigators recommend surgical intervention when the posterior fragment is measured to be one third to one fourth of the antero-posterior curvature of the articular surface of the tibial plafond [1-6]. Other authors support surgical intervention only when the fragment involves more than $50 \%$ of the articular surface [11]. Another common indication for surgery is a posterior malleolar fracture in combination with lateral malleolar fractures [12]. In general, if posterior subluxation of the tibiotalar joint is seen in the presence of a posterior malleolus fracture operative fixation is indicated. Finally, when the articular surface of the distal tibia is involved, or the integrity of the syndesmosis is jeopardized, surgical intervention should be considered [9, 10, 13]. When reduction and internal fixation of a posterior malleolar fracture are indicated, achieving anatomic fixation can be challenging. While medial and lateral malleolar fractures can be reduced anatomically during surgery, intraoperative fluoroscopic anterior posterior (AP), lateral, and mortise views generally underestimate the extent of the posterior malleolar fractures [12, 14]. Computed tomography $(\mathrm{CT})$ imaging significantly improved the accuracy of assessing the extent of posterior malleolar fractures [9] using Haraguchi et al.'s suggested classification $[12,14]$. Although some surgeons may consider it unnecessary, intraoperative CT [15] can be advantageous in the assessment of posterior malleolar reduction over fluoroscopic guidance alone. However, intraoperative CT is not preferred, or available, and preoperative CT imaging can be used for preoperative planning in primary cases. Some surgeons may employ early postoperative CT to assess the accuracy of their reduction.

Since 2009, the authors of this paper routinely performed early postoperative CT assessment of ankle fractures that involved posterior malleolar fractures after close or open reduction and internal fixation, in order to evaluate the accuracy of the reduction [16]. Despite the use of intraoperative fluoroscopy in order to achieve an acceptable reduction on image intensifier, many early postoperative CTs demonstrated an inaccurate reduction of the posterior malleolus in the eyes of the lead surgeon. In approximately two thirds of the cases reviewed, posterior malleolar fractures were under-reduced due to misplaced screws and poor understanding of the fracture pattern. It was previously shown that even limited posterior malleolar fragments are indicative of poor prognosis and portend an increased risk for the development of ankle arthritis [6, 14, 17-19]. In light of these findings, the objective of this study was to characterize posterior malleolar fractures based on preoperative X-ray using the standard Lauge-Hansen classification system [20], to characterize these fractures based on the location of the posterior malleolar fragments on preoperative CT imaging, and (3) to assess the posterior malleolar fracture different patterns to accurately optimized screw trajectory. Additionally, this study aimed to appraise the adequacy between the Lauge-Hansen classification system for predicting the appropriate screw trajectory in ankle fractures with a posterior malleolar component for each fracture's type.

\section{Materials and methods}

We performed a retrospective comparative cohort study of prospectively gathered data from a single center. The study was approved by the local institutional review board and took place between January 2014 and December 2016. A single fellowship-trained orthopedic surgeon performed all radiographic measurements. Eighty-five patients with posterior malleolar fractures were included in this study. Inclusion criteria were (1) skeletal maturity; (2) the presence of a posterior malleolar fragment that occupied one third or more of the antero-posterior curvature of the articular surface of the tibial plafond, the presence of a posterior malleolar fracture associated with tibiotalar dislocation, and non-reduced syndesmosis or the posterior inferior tibiofibular ligament (PITFL) which is connected to the posterior malleolar fragment; (3) the presence of a non-comminuted fracture without intra-articular fragments that would prevent screw fixation; (4) preoperative X-ray; (5) all the CT scan examinations which were performed during hospitalization; and (6) pre- and a post-operative CT scans which were performed within 1 week after internal fixation of the posterior malleolar fractures, in a case operative treatment was indicated. Pilon fracture of the distal tibia was excluded from the study.

In order to categorize the posterior malleolus fracture, two methods were used. In the first, we further developed the previously describe classification by Haraguchi 
et al. [14] which divided the posterior malleolus into 3 main types based on CT scan: posterolateral-oblique, small-shell type, and medial-extension which involves the medial malleolus in $20 \%$ of the cases. In our modified classification, the axial CT cut that demonstrated the largest posterior fragment was selected. The horizontal line was then drawn between the most prominent part of the medial malleolus and the Chaput tubercle, dividing the tibia into anterior and posterior segments. The posterior segment was then divided by a central line, perpendicular to the bimalleolar line, into medial and lateral sub-segments (Fig. 1a), thus creating a 4quadrant grid. Each posterior malleolar fracture was then categorized into one of the three groups based on the fragment's location: posterior fragments occupying predominantly the posterior-lateral sub-segment were classified as "postero-lateral" (not equivalent to a Volkmann fragment) (Fig. 1b), fragments occupying predominantly the posterior-medial segment were categorized as "postero-medial" (Fig. 1c), and fragments occupying both medial and lateral sub-segments were categorized as "postero-central" (Fig. 1d). The shape of the fragments was also noted.

Next, we used our superimposed images to plan and predict the optimal trajectory for posterior fragment fixation with a single posterior-to-anterior percutaneous screw. First, the most anterior prominent part of the tibial cortex located midway between the most prominent part of the medial malleolus and the Chaput tubercle on the lateral side of the tibia was the identified case on the CT cuts. These two points were chosen because, in practice, they are easily found by palpation and can therefore be used intraoperatively. Second, a reference line was marked perpendicular to the bimalleolar line. Third, a trajectory line, perpendicular to the fracture line, was marked from the most prominent anterior part toward
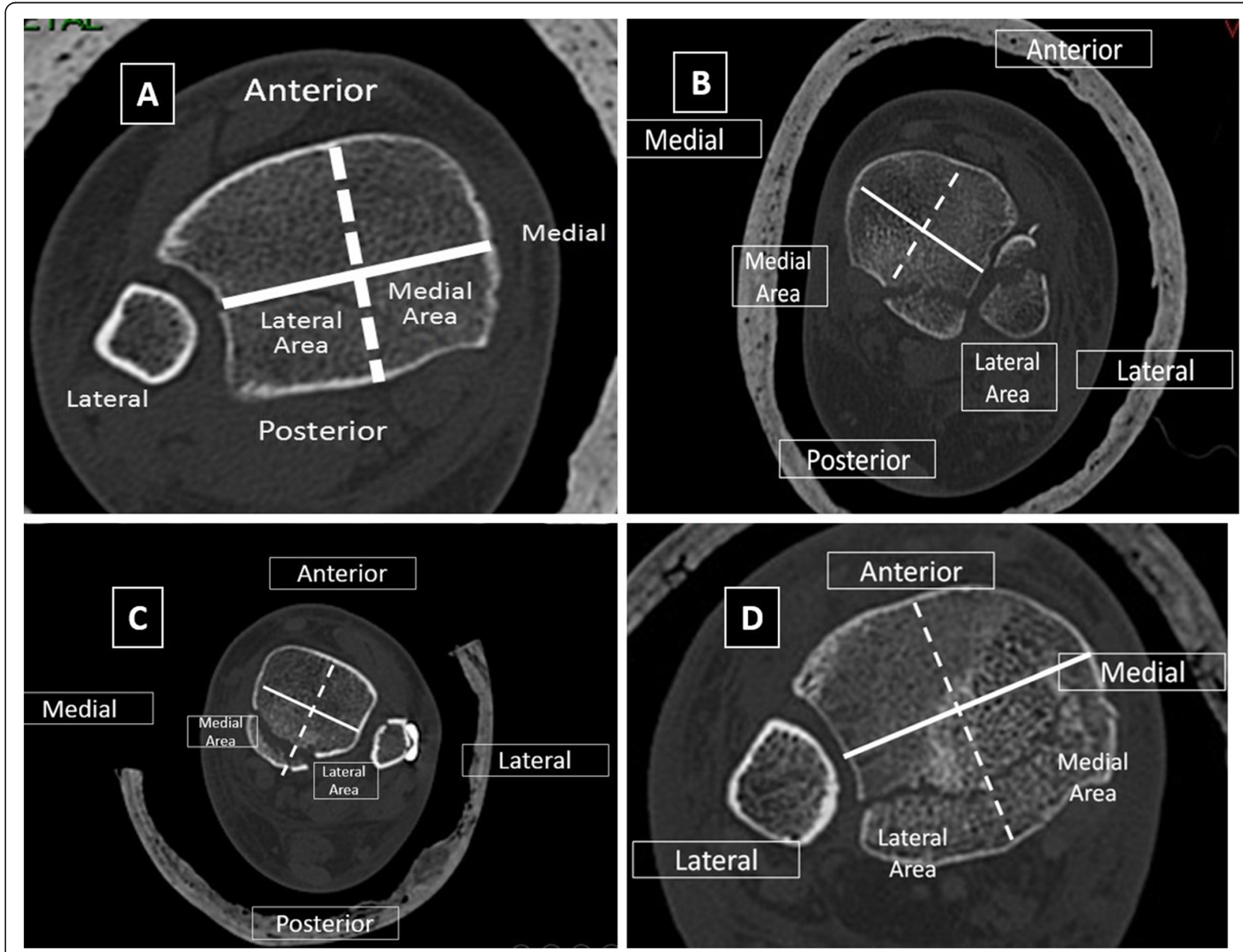

Fig. 1 A method for CT-based classification of posterior malleolar fractures. a Superimposed on an axial CT image of the ankle, a solid line is subtended between the medial and lateral malleoli. Then, a second (dashed) line is drawn perpendicularly to the first intermalleolar line, dividing the posterior malleolus area into medial and lateral segments. b- $\mathbf{d}$ CT images showing the different fracture types: postero-lateral (b), posteromedial (c), and postero-central (d) 
the posterior part of the fragment on the axial CT images. The angle formed between this line and the line perpendicular to the bimalleolar line was defined as the "trajectory angle" (Fig. 2). This angle was measured and averaged for each fragment type.

The second classification method we used was according to the Lauge-Hansen classification system [20] and the Arimoto and Forrester ankle fracture algorithm [21]. All was done by one investigator, based on standard trauma series radiographs.

\section{Surgical technique}

In order to obtain optimized screw trajectory for internal fixation based on fragment classification (i.e., "posterolateral," "postero-medial," or "postero-central"), a reference Kirschner wire (rKW) was first inserted from the most anteriorly prominent and palpable part on the tibial cortex, midway between the medial prominence of the medial malleolus and the Chaput tubercle, in an anterior to posterior direction under antero-posterior fluoroscopic imaging (Fig. 2). Using lateral fluoroscopic imaging, the rKW was then advanced posteriorly while maintained parallel to the ankle joint line. The trajectory Kirschner wire (tKW) was then inserted adjacent to the $\mathrm{rKW}$, at an angle corresponding to the mean trajectory angle for the specific morphological type of the posterior malleolar fragment, parallel to the joint line (Fig. 3). A posterior-to-anterior-cannulated screw was then inserted over the $\mathrm{tKW}$ in order to stabilize the posterior fragment
(Fig. 4). It is advisable to make a small skin incision followed by a minimal blunt dissection prior to the KW and screw insertion, based on the planned hardware route, in order to avoid damaging the surrounding neuro-vascular structures.

\section{Statistical analysis}

Descriptive statistics are presented as mean values with ranges. Student $t$ tests were used to examine the differences between the trajectory angles among each of the three CT-based fracture classification groups and for each of the Lauge-Hansen fracture types. An alpha error of 0.05 was considered statistically significant.

\section{Results}

A total of 85 patients who underwent surgical internal fixation for a posterior malleolar fracture between January 2014 and December 2016 met all the inclusion criteria for this study. This included 62 females and 23 males with a mean age of 53.4 years (range, 17-89 years). In $60 \%$ of cases (51 ankles), the right ankle was involved. Sixty-one cases were classified as "postero-lateral," four cases as "postero-medial," and 20 cases as "postero-central," with mean trajectory angles of $21^{\circ}\left(17-26^{\circ}\right), 28^{\circ}$ $\left(25-31^{\circ}\right)$, and $7^{\circ}\left(2-13^{\circ}\right)$, respectively. The measurements are summarized in Table 1 . Trajectory angles were significantly different between all the fracture types $(p<$ 0.01 ). More than $60 \%$ of the cases were either supination-lateral rotation type IV, or pronation-

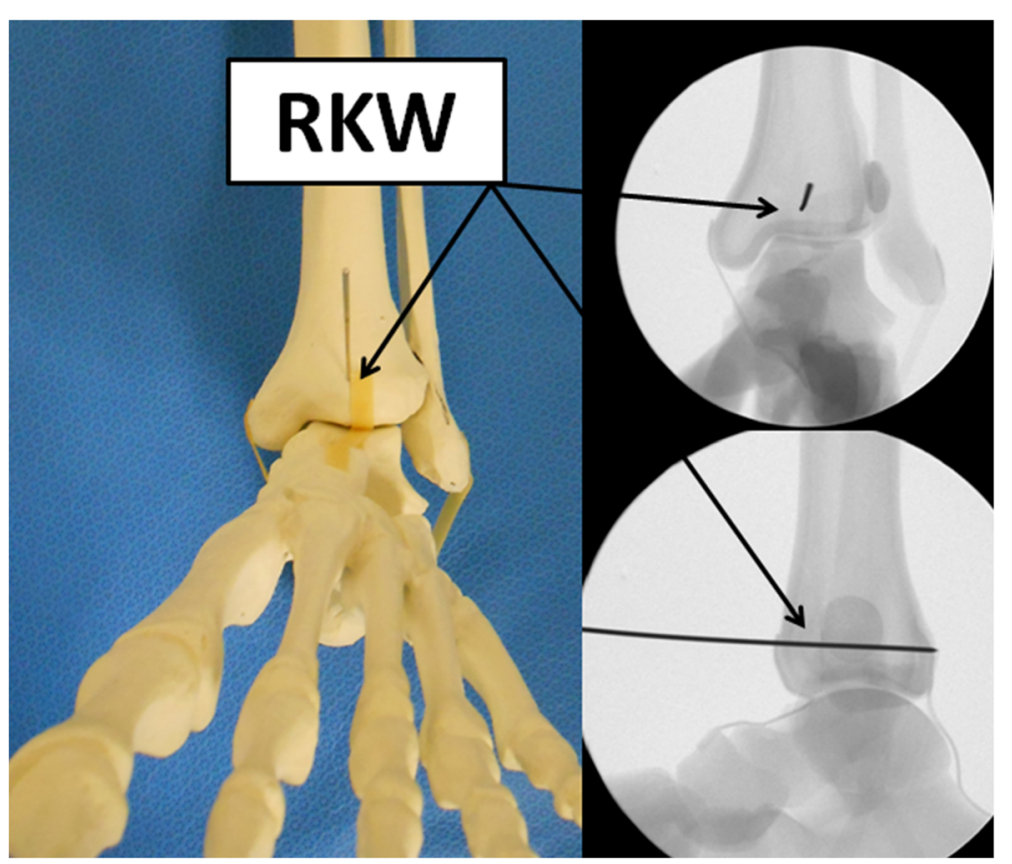

Fig. 2 A reference K-wire (rKW) is inserted midway between the medial malleolus and the Chaput prominence, parallel to the articular surface in antero-posterior direction 


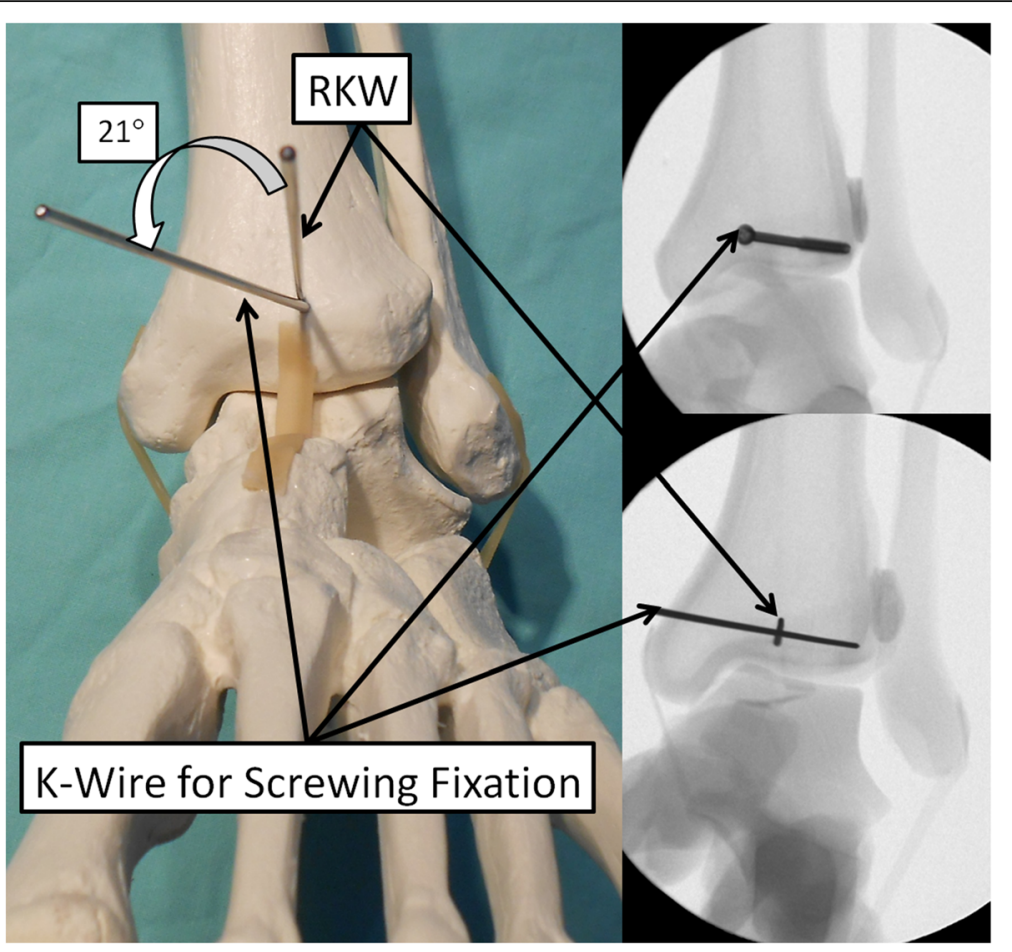

Fig. 3 A second K-wire is inserted as a guide-pin for the screw in the correct trajectory angle in order to intersect and adequately stabilize the posterior malleolar fragment. The direction of this K-wire corresponds to the mean trajectory angle of the specific fracture type calculated on the axial CT cut

abduction type III, when classified according to the Lauge-Hansen classification (Table 2). No statistically significant differences were found comparing the average trajectory angle among each of the Lauge-Hansen groups $(p>0.05)$. Figure 4 shows an example of a "postero-lateral" type fracture which was stabilized with a cannulated screw using the described surgical technique, with a mean trajectory angle of $21^{\circ}$.

\section{Discussion}

The primary outcome of this study is a set of average trajectory angles for an ideal single percutaneous posterior-to-anterior lag screw fixation of posterior malleolar fractures, based on pre-operative axial CT images. The impetus for this study stems from the anecdotal and scientific evidence which demonstrates unsatisfactory functional outcomes in patients with ankle fractures including a posterior malleolar fragment $[6,14,17]$. In some cases, even limited posterior malleolar fragments are indicative of poor prognosis and portend an increased risk for the development of ankle arthritis [18, $19,22]$. While failure of treatment is commonly multifactorial, in cases of posterior malleolar fractures, a substantial contributing factor to inferior outcomes and the progression of ankle arthritis may be the difficulty in obtaining anatomic close reduction of the posterior malleolar fragment. This may result in tibiotalar joint incongruity, which substantially increases joint contact pressure [13, 22, 23]. Moreover, mal-positioned screws in a posterior malleolar fracture could inadvertently cause mal-reduction of the syndesmosis and lead to separation of the fibula from the anatomical location in the incisura [10, 13, 16]. Fitzpatrick et al. [10] conducted a cadaveric study of 9 specimens with a supinationexternal rotation injury with a posterior malleolus fracture. The authors showed a direct link between syndesmotic mal-reduction and posterior malleolus malreduction. Screws that entirely miss the posterior malleolar fragment or do not capture the fragment well can lead to joint incongruencies or subluxation. As the extent and location of posterior malleolar fractures are optimally assessed on axial CT cuts and are commonly miss-appreciated on standard radiographs [12, 14]. We have presented how a preoperative CT scan can be used in order to avoid these hazardous surgical pitfalls.

As previously discussed in the introduction part, indications for surgery in cases of posterior malleolar fractures are inconclusive and usually rely on the size of the posterior malleolar fragment and the presence of posterior subluxation of the tibial-talar joint [1-6]. The extent and location of posterior malleolar fractures are optimally assessed on axial CT cuts and are commonly misappreciated on standard radiographs ${ }^{12 ;} 14 ; 24 ; 25$. Thus, reducing and fixing these fragments anatomically using 


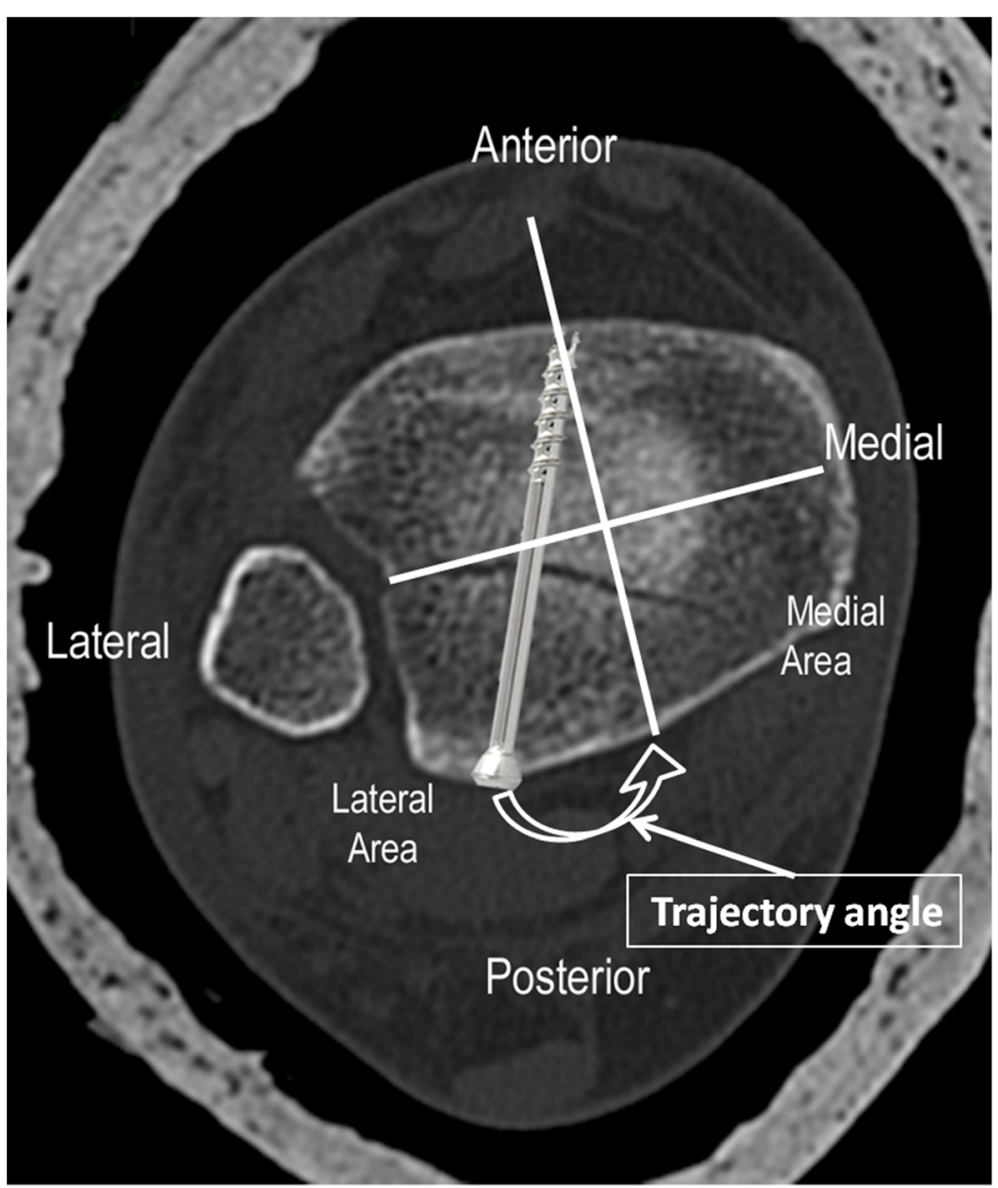

Fig. 4 Axial CT image demonstrating the trajectory angle and ideal screw lag placement in postero-lateral type posterior malleolar fracture

only fluoroscopic-guided surgery becomes challenging. Nevertheless, it is not common for surgeons to use intra-operative CT in these cases [15]. Solan et al. [13] also advocated the use of a CT scan for a better understanding of the patterns of posterior malleolus fractures that extend medially and to ensure adequate reduction.

Previously, there have been attempts to categorize ankle fractures with a posterior fragment using CT imaging. Bartoníček et al. [24] divided the fractures into five categories, based on the size, shape, location of the

Table 1 Trajectory angles in each of the CT-based posterior malleolar fracture types

\begin{tabular}{lll}
\hline Fracture type & Number (\%) of cases & Mean TA (range) $\left(^{\circ}\right)$ \\
\hline Postero-medial & $4(4.7 \%)$ & $28(25-31)$ \\
Postero-central & $20(23.5 \%)$ & $7(2-13)$ \\
Postero-lateral & $61(71.8 \%)$ & $21(17-26)$ \\
\hline
\end{tabular}

For "postero-medial" fragments, the trajectory angle line is typically subtended from a starting point medial to the AP central line. For "postero-lateral" and "postero-central" fragments, the trajectory angle line is typically subtended from a starting point lateral to the AP central line $T A$ trajectory angle fragment, stability of the tibiotalar joint, and the integrity of the fibular notch. A large number of types may be regarded as a disadvantage from a clinical perspective. In addition, the authors include in the analysis pilon fractures, which have a very different pathomechanism. Mason et al. [25] divided the fractures into 3 groups based on severity and pathomechanics. Type 1 is an extra-articular posterior malleolar fragment, type 2 involves a primary fragment of the Volkman area with or without a medial injury, and type 3 is a fracture in the coronal plane that includes all the plafond. The medial posterior fracture group in our series did not match any of these types.

Haraguchi et al. [14] were the first to divide and classified the posterior malleolus fracture based on a CT scan of 57 patients. The authors introduce 3 common patterns: posterolateral oblique which constituted $38 \%$ of all the types, medial extension (19\%), and small-shell type (14\%). The aim of the study was mainly descriptive to examine the ratio of the posterior fragment area to the total crosssectional area of the tibial plafond, along with the angle between the bimalleolar axis and the major fracture line. 
Table 2 Trajectory angles in each of the Lauge-Hansen classification-based posterior malleolar fracture types

\begin{tabular}{lll}
\hline Fracture type & Number (\%) of cases & Mean TA (range) $\left(^{\circ}\right)$ \\
\hline Pronation abduction injury-type II & $5(5.9 \%)$ & $18.4(5.4-22.7)$ \\
Pronation abduction injury—type III & $20(23.5 \%)$ & $19.4(3.5-30.9)$ \\
Pronation lateral rotation injury—type IV & $13(15.3 \%)$ & $20.7(10.8-31.7)$ \\
Supination abduction injury—type II & $1(1.2 \%)$ & 3 \\
Supination lateral rotation injury—type III & $8(9.4 \%)$ & $17.7(2.1-22.9)$ \\
Supination lateral rotation injury_type IV & $32(37.6 \%)$ & $18.1(2.1-26.7)$ \\
Isolated posterior lip injury & $6(7.1 \%)$ & $16.3(4.4-22.3)$ \\
\hline
\end{tabular}

TA trajectory angle

In our study, we examined 85 CT scans, and based on Haraguchi et al., we further developed the classification and found 3 different fracture patterns with distinct mean trajectory angles which may be applicable in the operation theater using merely an intraoperative fluoroscopy. Furthermore, we compared the Lauge-Hansen classification system [20] to the posterior malleolus fracture pattern has seen on a CT scan and found it to be incompatible.

The Lauge-Hansen classification system for ankle fractures is commonly used in orthopedic trauma surgery and is based solely on plain radiographs. The classification was designed to describe the mechanism of injury; however, low accuracy demonstrated with this classification [7]. This study did not find a good correlation between the ideal trajectory angles and the Lauge-Hansen classification of each ankle fracture type. The mean trajectory angle by Lauge-Hansen classification was approximately $20^{\circ}$, demonstrating that the classification does not have a sensitivity to distinguish between different posterior malleolar fracture patterns. A preoperative or intraoperative CT scan can greatly assist in the anatomic articular reduction in these cases. Furthermore, the Lauge-Hansen classification does not differentiate nor describe the different patterns of the correlates with the area of the posterior malleoli fracture and thus does not correlate with the findings and the suggested modified classification of this study.

Utilizing axial CT cuts, we were able to categorize each posterior malleolar fracture into one of three major categories. The most common type was "postero-lateral, " which comprised about two thirds of the cases. This is consistent with a previous study, which showed that the most frequent type of posterior malleolus ankle fractures involved the postero-lateral part of the malleolus [14]. The mean trajectory angle required for properly intersecting and fixing this type of fragment was found to be approximately $21^{\circ}\left(17-26^{\circ}\right)$ in this series. Therefore, using a trajectory angle of $20^{\circ}$ aiming laterally from the central antero-posterior line during surgery and using the antero-posterior and lateral fluoroscopic views as described may increase the odds of obtaining good fixation in the majority of "postero-lateral" fragments.
For "postero-medial" and "postero-central" types, the range of trajectory angles needed for a proper posterior malleolar fragment fixation was also relatively narrow. A trajectory angle of approximately $28^{\circ}$ aiming medially from the central antero-posterior line may capture the majority of "postero-medial" fragments, and a trajectory angle of approximately $7^{\circ}$ aiming laterally from the central antero-posterior line may capture the majority of "postero-central" fractures. As the mean trajectory angles for all three types are relatively reproducible, they may be a good intraoperative reference for surgeons to use in addition to fluoroscopic reduction and their primary ankle fracture fixation techniques. However, cautions should be used when applying this technique since in some situations, two posterior-to-anterior lag screws may be preferred, or a single screw in addition to a posterior buttress plate may be superior to a single lag screw. The mean trajectory angles that are provided in this paper are meant to guide a surgeon's understanding of common fracture patterns and not to suggest that these fractures should ideally be fixed with a single anterior-to-posterior lag screw in any or all cases.

Although the presented categorization system could describe most posterior malleolus fractures in our series, four cases were comminuted and did not fit in any category. This is consistent with previous descriptions of posterior malleolar fractures [14] and implies that using the CT-based trajectory angle as described here may not be sufficient to provide anatomic reduction and mechanical stability of all fractures. In these cases, posterior buttress plating may be more appropriate than using screws alone. When the fragmented area is medial, the interval between the flexor hallucis longus and the Achilles tendon can be used for optimal surgical approach, and when the fragmented area is lateral, the interval between the peroneal tendons and the Achilles can be used. Moreover, combinations of "postero-lateral" and "postero-medial" fragments can occur in posterior malleolar fractures [14]. In these cases, it may be optimal to use at least two lag screws at the appropriate trajectory angles or posterior buttress plating. 


\section{Study limitations}

The limitations of this study include the lack of clinical outcome data to confirm the reproducibility of the surgical technique described. Furthermore, the cost and implications of increased radiation associated with $\mathrm{CT}$ imaging of all intraarticular ankle fractures are not insignificant. The different trajectory angles and their proximity to the surrounding neuro-vascular structures were not thoroughly examined in practice, and further investigation is needed. Nonetheless, we assumed a small skin incision followed by a minimal blunt dissection prior to the KW and screw insertion would provide sufficient protraction. Prospective controlled trials of different fixation methods and imaging techniques that include assessment of patient-reported clinical outcomes would further illuminate the effectiveness of different approaches to posterior malleolar fragment fixation.

Conclusion This study defines three distinct and common anatomic subgroups of posterior malleolar fragments and defines the average trajectory angle for ideal posterior to anterior lag screw fixation of these subgroups. The use of CT imaging in intraarticular ankle fractures and specifically in posterior malleolar fractures can increase the understanding of this fracture pattern with the goal of improving anatomic fixation and ultimately, the clinical outcomes. Moreover, the LaugeHansen classification system may be inadequate to characterize specific variants of posterior malleolar fracture patterns and this should be taken into account during operative fixation of ankle fractures.

\section{Abbreviations}

AP: Antero-posterior; CT: Computed tomography; rKW: Reference Kirschner Wire; tKW: Trajectory Kirschner Wire

\section{Acknowledgements}

We thank Mr. Nitzan Konstantin for assistance with writing the manuscript and to Prof. Meir Nyska for helping in the planning of the paper and the review.

\section{Authors' contributions}

OS and DS collected the data. EP and EY analyzed and interpreted the X-ray images. EP, ZTS, and MV were the major contributors in writing the manuscript. NO and RA reviewed the manuscript. All authors read and approved the final manuscript.

\section{Funding}

Not applicable.

\section{Availability of data and materials}

Not applicable.

\section{Ethics approval and consent to participate}

The study was approved by the local institutional review board.

\section{Consent for publication}

Not applicable.

\section{Competing interests}

The authors declare that they have no competing interests.

\section{Author details}

'Orthopedic Department, Meir Hospital, Sapir Medical Center affiliated with the Sackler Faculty of Medicine, Tel Aviv University, 56 Tchernichovsky St, Kfar Saba, Israel. ${ }^{2}$ Department of Orthopaedic Surgery, Montefiore Medical Center, Albert Einstein College of Medicine, 1300 Morris Park Ave, The Bronx, NY 10461, USA. ${ }^{3}$ Department of Orthopaedic Surgery, Tel Aviv Medical Center affiliated with the Sackler Faculty of Medicine, Tel Aviv University, Weizmann St. 6, Tel Aviv-Yafo, Israel. ${ }^{4}$ Department of Orthopaedic Surgery, Assuta Medical Center affiliated with the Faculty of Health and Science, Ben Gurion University of the Negev, Menachem Begin Blvd. 126, Ashdod, Israel.

Received: 10 August 2019 Accepted: 16 March 2020

Published online: 26 March 2020

\section{References}

1. Vander Griend R, Michelson JD, Bone LB. Fractures of the ankle and the distal part of the tibia. Instr Course Lect. 1997;46:311-21.

2. Lash $\mathrm{N}$, Horne $\mathrm{G}$, Fielden J, et al. Ankle fractures: functional and lifestyle outcomes at 2 years. ANZ journal of surgery. 2002;72:724-30.

3. Court-Brown CM, McBirnie J, Wilson G. Adult ankle fractures--an increasing problem? Acta orthopaedica Scandinavica. 1998;69:43-7.

4. Daly PJ, Fitzgerald RH Jr, Melton $\sqcup$, et al. Epidemiology of ankle fractures in Rochester, Minnesota. Acta Orthop Scand. 1987;58:539-44.

5. Broos PL, Bisschop AP. Operative treatment of ankle fractures in adults: correlation between types of fracture and final results. Injury. 1991;22:403-6.

6. Jaskulka RA, Ittner $G$, Schedl R. Fractures of the posterior tibial margin: their role in the prognosis of malleolar fractures. The Journal of trauma. 1989;29: 1565-70.

7. Kwon JY, Chacko AT, Kadzielski JJ, et al. A novel methodology for the study of injury mechanism: ankle fracture analysis using injury videos posted on YouTube.com. Journal of orthopaedic trauma. 2010;24:477-82.

8. Hak DJ, Egol KA, Gardner MJ, et al. The "not so simple" ankle fracture: avoiding problems and pitfalls to improve patient outcomes. Instr Course Lect. 2011;60:73-88.

9. Lampridis V, Gougoulias N, Sakellariou A. Stability in ankle fractures: diagnosis and treatment. EFORT Open Rev. 2018;3:294-303.

10. Fitzpatrick E, Goetz JE, Sittapairoj T, et al. Effect of posterior malleolus fracture on syndesmotic reduction: a cadaveric study. The Journal of bone and joint surgery American volume. 2018;100:243-8.

11. Gardner MJ, Streubel PN, McCormick JJ, et al. Surgeon practices regarding operative treatment of posterior malleolus fractures. Foot Ankle Int. 2011;32: 385-93.

12. Palmanovich $E$, Brin $Y S$, Laver $L$, et al. The effect of minimally displaced posterior malleolar fractures on decision making in minimally displaced lateral malleolus fractures. Int Orthop. 2014;38:1051-6.

13. Solan MC, Sakellariou A. 2017. Posterior malleolus fractures: worth fixing The bone \& joint journal 99-b:1413-1419.

14. Haraguchi $\mathrm{N}$, Haruyama $\mathrm{H}$, Toga $\mathrm{H}$, et al. Pathoanatomy of posterior malleolar fractures of the ankle. The Journal of bone and joint surgery American volume. 2006;88:1085-92.

15. Hsu AR, Gross CE, Lee S. Intraoperative O-arm computed tomography evaluation of syndesmotic reduction: case report. Foot Ankle Int. 2013;34: 753-9.

16. Palmanovich E, Brin YS, Kish B, et al. Value of early postoperative computed tomography assessment in ankle fractures defining joint congruity and criticizing the need for early revision surgery. J Foot Ankle Surg. 2016;55: 465-9.

17. McDaniel WJ, Wilson FC. Trimalleolar fractures of the ankle. An end result study. Clin Orthop Related Res. 1977:37-45.

18. Thomas RH, Daniels TR. Ankle arthritis. The Journal of bone and joint surgery American volume. 2003;85:923-36.

19. Macko WW, Matthews LS, Zwirkoski P, et al. The joint-contact area of the ankle. The contribution of the posterior malleolus. J Bone Joint Surg Am Vol. 1991;73:347-51.

20. Lauge-Hansen N. Ligamentous ankle fractures; diagnosis and treatment. Acta chirurgica Scandinavica. 1949;97:544-50.

21. Arimoto HK, Forrester DM. Classification of ankle fractures: an algorithm. AJR American journal of roentgenology. 1980;135:1057-63.

22. van den Bekerom MP, van Dijk CN. Is fibular fracture displacement consistent with tibiotalar displacement? Clinical orthopaedics and related research. 2010;468:969-74. 
23. Lloyd J, Elsayed S, Hariharan K, et al. Revisiting the concept of talar shift in ankle fractures. Foot Ankle Int. 2006;27:793-6.

24. Bartonicek J, Rammelt S, Kostlivy K, et al. Anatomy and classification of the posterior tibial fragment in ankle fractures. Archives of orthopaedic and trauma surgery. 2015;135:505-16.

25. Mason LW, Marlow WJ, Widnall J, et al. Pathoanatomy and associated injuries of posterior malleolus fracture of the ankle. Foot Ankle Int. 2017;38: 1229-35.

\section{Publisher's Note}

Springer Nature remains neutral with regard to jurisdictional claims in published maps and institutional affiliations.

Ready to submit your research? Choose BMC and benefit from:

- fast, convenient online submission

- thorough peer review by experienced researchers in your field

- rapid publication on acceptance

- support for research data, including large and complex data types

- gold Open Access which fosters wider collaboration and increased citations

- maximum visibility for your research: over $100 \mathrm{M}$ website views per year

At BMC, research is always in progress.

Learn more biomedcentral.com/submissions 